\title{
RNA sequencing uncovers the key long non-coding RNAs and potential molecular mechanism contributing to XAV939-mediated inhibition of non-small cell lung cancer
}

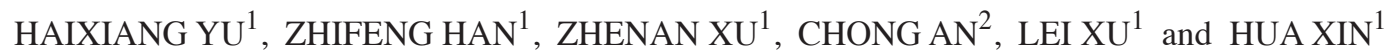 \\ ${ }^{1}$ Department of Thoracic Surgery, China-Japan Union Hospital of Jilin University, Changchun, Jilin 130033; \\ ${ }^{2}$ Department of Thoracic Surgery, The Second Hospital of Jilin City, Jilin 132001, P.R. China
}

Received May 31, 2018; Accepted February 14, 2019

DOI: $10.3892 / \mathrm{ol} .2019 .10191$

\begin{abstract}
The present study aimed to reveal the key long non-coding RNAs (lncRNAs) and the potential molecular mechanisms of XAV939 treatment in non-small cell lung cancer (NSCLC). The NSCLC cell line, NCI-H1299, was cultured with $10 \mu \mathrm{M}$ XAV939 for $12 \mathrm{~h}$, and NCI-H1299 cells without XAV939 treatment were used as controls. Following RNA isolation from the two groups, RNA-sequencing was performed to detect transcript expression levels, and differentially-expressed lncRNAs (DE-lncRNAs) and DE-genes (DEGs) were identified between groups and analyzed for their functions and associated pathways. The potential associations between proteins encoded by DEGs were revealed via a protein-protein interaction (PPI) network. Subsequently, the microRNA (miRNA/miR)-mRNA, lncRNA-miRNA and lncRNA-mRNA interactions were explored, followed by competing endogenous RNA (ceRNA) network construction. A total of 396 DEGs and 224 DE-lncRNAs were identified between the XAV939 and control groups. These lncRNAs were mainly enriched in pathways such as 'ferroptosis' [DEG, solute carrier family 7 member 11 (SLC7A11)]. The PPI network consisted of 97 nodes and 112 interactions. Furthermore, a total of 10 noteworthy lncRNAs
\end{abstract}

Correspondence to: Dr Lei Xu or Dr Hua Xin, Department of Thoracic Surgery, China-Japan Union Hospital of Jilin University, 126 Xiantai Street, Erdao, Changchun, Jilin 130033, P.R. China

E-mail: xieliang767zhui@163.com

E-mail: 13596118509@163.com

Abbreviations: lncRNAs, long non-coding RNAs; NSCLC, non-small cell lung cancer; DE-lncRNA, differentially-expressed lncRNA; PPI, protein-protein interaction; ceRNA, competing endogenous RNA; GO, Gene Ontology; KEGG, Kyoto Encyclopedia of Genes and Genomes; MF, molecular function; CC, cellular componenT; ST3GAL6, ST3 $\beta$-galactosile $\alpha$-2,3-sialyltransferase 6; AS1, antisense RNA 1; SLF, solute carrier family

Key words: non-small cell lung cancer, XAV939, RNA sequencing, long non-coding RNAs, competing endogenous RNA network, function and pathway analysis were revealed in the DE-lncRNA-DEG interaction. Finally, the IncRNA-miRNA-mRNA regulatory association, including MIR503 host gene (MIR503HG)-miR1273c-SRY-box 4 (SOX4), was explored in the current ceRNA network. The downregulation of lncRNA MIR503HG induced by XAV939 may serve an important role in NSCLC suppression via sponging $m i R-1273 \mathrm{c}$ and regulating SOX4 expression. Furthermore, the downregulation of SLC7A11 induced by XAV939 may also inhibit the development of NSCLC via the ferroptosis pathway.

\section{Introduction}

Lung cancer occurs from uncontrolled cell growth in the lung tissue, resulting in tumors (1). Non-small cell lung cancer (NSCLC) is one of the most common types of lung tumor (2). The 5-year survival rate across all stages of NSCLC is only $12 \%$ (3). Unfortunately, the median survival time for NSCLC patients is $<1$ year following diagnosis (4). Thus, improved outcomes for NSCLC are clearly required. The improvement of clinical outcomes for NSCLC patients can be determined by a deep investigation of the molecular mechanisms (5). Previous studies have shown that abnormal expression of long non-coding RNAs (lncRNAs) is strongly associated with the pathogenesis of NSCLC (6,7). Differentially expressed lncRNAs (DE-lncRNAs), including HOX transcript antisense RNA and metastasis-associated lung adenocarcinoma transcript 1 , serve important roles in the progression of NSCLC via the epidermal growth factor receptor-tyrosine kinase inhibitor resistance pathway (6). As a potential biomarker, the lncRNA signature contributes to the survival prediction of NSCLC patients (8). Ghadimi et al (9) showed that mRNAs, including lung-specific $\mathrm{X}$ protein $(L U N X)$ and carcinoembryonic antigen, in the pleural fluid can serve as promising biomarkers for the detection of NSCLC (9). The plasma LUNX mRNA is another non-invasive specific biomarker for the diagnosis and prognostic prediction of NSCLC (10). Apart from mRNAs, microRNAs (miRNAs/miRs) are commonly used to identify novel NSCLC genes and their associated networks. A previous study showed that miRNAs, including $m i R-98-5 p$ and $m i R-302 e$, can be used as markers for predicting radio-sensitivity in NSCLC (11). The IncRNA-miRNA-mRNA regulatory network, known as the competing endogenous RNA (ceRNA) 
network, is an important tripartite axis in the regulation of the disease process $(12,13)$. Although the identification of molecular factors contributes to therapeutic interventions in NSCLC, the key lncRNAs and the possible regulatory mechanism of ceRNAs in NSCLC remains unclear.

Recent technological advances in high-throughput gene sequencing and expression profiling have allowed the analysis of gene expression in NSCLC $(14,15)$. As a small molecular inhibitor, XAV939 promotes apoptosis in the tumor cell line via telomere shortening (16). A previous study showed that XAV939 can inhibit the proliferation and migration of lung adenocarcinoma cells (17). In the present study, high-throughput sequencing was performed on XAV939-treated NCI-H1299 cells (XAV939 group) and untreated controls (control group), to explore the DE-lncRNAs and DEGs. Subsequently, a protein-protein interaction (PPI) network was constructed for DEG-encoded proteins. The associated functions of DEGs and DE-lncRNAs were further explored. The miRNA-DEG associations were subsequently explored, followed by investigation of the miRNA-DEG regulatory network. Finally, the ceRNA network was constructed and analyzed. The present study attempted to explore the potential mechanism of NSCLC and provide information regarding novel genes as potential targets for the treatment of NSCLC.

\section{Materials and methods}

Cell culture. The NSCLC cell line, NCI-H1299, was purchased from the Type Culture Collection of the Chinese Academy of Sciences (Shanghai, China) and maintained in RPMI-1640 medium (Gibco; Thermo Fisher Scientific, Inc., Waltham, MA, USA) supplemented with $10 \%$ fetal bovine serum (FBS; Gibco; Thermo Fisher Scientific, Inc.) and $1 \%$ penicillin-streptomycin (Sangon Biotech Co., Ltd., Shanghai, China) in a humidified incubator with a $5 \% \mathrm{CO}_{2}$ atmosphere at $37^{\circ} \mathrm{C}$.

Cell Counting Kit-8 (CCK-8) assay. Following XAV939 (Sigma-Aldrich; Merck KGaA, Darmstadt, Germany) treatment, CCK-8 (Beyotime Institute of Biotechnology, Shanghai, China) assay was used to determine the viability of the NCI-H1299 cells. The NCI-H1299 cells were seeded in a 96 -well plate at a density of $5.0 \times 10^{3}$ per well overnight. Subsequently, the cells were treated with low and high concentrations of XAV939 (10 and $20 \mu \mathrm{M} / \mathrm{ml}$, respectively) for $24 \mathrm{~h}$ and NCI-H1299 cells without XAV939 were used as the control group, with 5 repeats in each group. The doses and time point selected were used as referenced by previous studies (17-19). In addition, the NCI-H1299 cell morphology was observed following treatemn with XAV939 under a light microscope (YYS-190E; Shanghai Optical Instrument Co., Ltd., Shanghai, China). Following $22 \mathrm{~h}$ of treatment, $10 \mu \mathrm{l}$ CCK-8 solution (Beyotime Institute of Biotechnology) was added to each well and incubated for $2 \mathrm{~h}$. Subsequently, the optical density (OD) of each well at $450 \mathrm{~nm}$ was determined using the Infinite M100 PRO (Tecan Group Ltd., Mannedorf, Switzerland).

Apoptosis analysis. The NCI-H1299 cells were seeded in a 6 -well plate at a density of $1 \times 10^{5}$ cells $/ \mathrm{ml}$ overnight. On the 2nd day, the NCI-H1299 cells were treated with $10 \mu \mathrm{M}$ XAV939 (Sigma-Aldrich) and the NCI-H1299 cells without treatment were used as the control group. Following treatment for $48 \mathrm{~h}$, the NCI-H1299 cells from the treatment and the control groups were washed with PBS and incubated in $1 \mathrm{ml}$ PBS containing $100 \mu \mathrm{l} 1 \mathrm{X}$ binding buffer, $5 \mu \mathrm{l}$ propidium iodide (Sigma-Aldrich) and $5 \mu \mathrm{l}$ FITC-Annexin-V (BD Biosciences, San Jose, CA, USA) in the dark, at room temperature for $15 \mathrm{~min}$. Finally, $400 \mu \mathrm{l} 1 \mathrm{X}$ binding buffer was added to each well and the FACSCalibur flow cytometer (BD Biosciences) was used to analyze apoptosis within $1 \mathrm{~h}$ and data were analyzed with FlowJo version 10 software (FlowJo LLC, Ashland, OR, USA).

Cell preparation for RNA-sequencing (RNA-Seq). The NCI-H1299 cells in the treatment group, including three samples termed s1, s2 and s3, at confluence were cultured in a 12-well plate supplemented with $10 \mu \mathrm{M}$ XAV939 for $24 \mathrm{~h}$ (XAV939 group), while those without XAV939 treatment were used as controls (control group; including three samples termed k1, k2 and k3). Following $24 \mathrm{~h}$ of culture, the six cell samples in the 12-well plate were washed with PBS and the total RNA of samples were extracted for RNA-seq.

RNA isolation and RNA-Seq. The six RNA samples from the two groups were isolated using a TRIzol ${ }^{\circledR}$ kit (Takara Biotechnology Co., Ltd., Dalian, China). The total RNA was quantified with a NanoDrop 2000 (Thermo Fisher Scientific, Inc.). Subsequently, total RNA (3 $\mu \mathrm{g} / \mathrm{sample})$ was reverse-transcribed to construct a cDNA library using a NEBNext ${ }^{\circledR}$ Ultra $^{\mathrm{TM}}$ RNA Library Prep kit for Illumina ${ }^{\circledR}$ (cat no. E7530L; New England Biolabs, Inc., Ipswich, MA, USA) according to the manufacturer's protocol. mRNAs were enriched on a magnetic bead prior to shearing into fragments. Following this, the cDNAs were synthesized and amplified with 15 cycles of the repair chain reaction. The cDNA clusters were sequenced using the Illumina HiSeq 4000 platform (Illumina, Inc., San Diego, CA, USA) using the 150 paired end method (20).

Pretreatment of RNA-seq data. Quality control was performed with Trimmomatic tools (v3.6) (21) as follows: i) Removal of the barcode sequences, ii) elimination of the base at each end with a quality $<10$, iii) exclusion of low-quality reads in which bases with quality $>20$ accounted for $<80 \%$ of the length and iv) exclusion of reads with a length $<50$ nucelotides. Clean reads from all the samples were obtained and aligned with the human reference genome (GRCh38.p7) (22) using TopHat software (v2.1.0) (23) with the default parameters.

Gene expression levels analysis. Following pretreatment of the raw RNA-seq data, gene expression levels were determined by counting reads in the genomic region or the exon region. The read count was obtained based on human genome annotation information in the GENCODE database (v25; https://www.gencodegenes.org/human/releases.html) using the featureCounts software (v1.6.2; http://subread.sourceforge. net/), followed by normalization based on the reads per kilobase per million (RPKM) mapped reads method. Genes with a RPKM value $<0.1$ in $\geq 3$ samples were defined as having low expression. Genes were classified as lncRNA or mRNA based on the annotation information. 

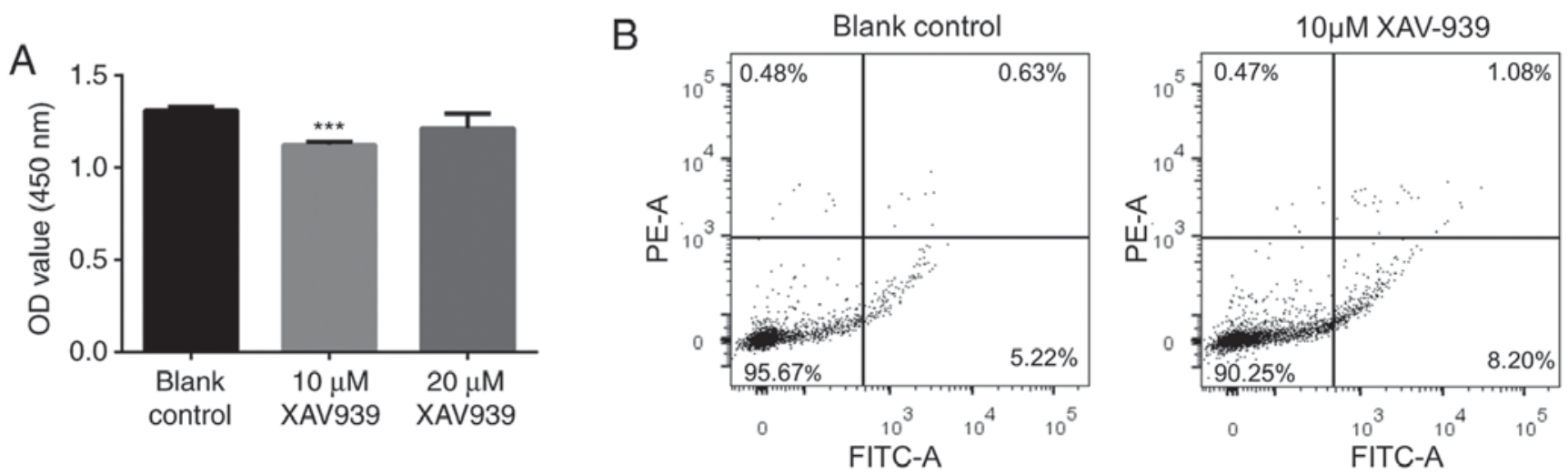

Figure 1. XAV939 treatment decreases proliferation, but increases apoptosis of NCI-H1299 cells. (A) Proliferation of the NCI-H1299 cells between the control group and NCI-H1299 cells treated with either 10 or $20 \mu \mathrm{M}$ XAV939, determined by Cell Counting Kit- $8 .{ }^{* * *} \mathrm{P}<0.001$, compared with the control group. (B) Apoptosis analysis of the NCI-H1299 cells determined by flow cytometry to compare the control group and $10 \mu$ M XAV939-treatment group. OD, optical density; PE-A, phycoerythrin; FITC-A, fluorescein isothiocyanate.

Identification of DEGs and DE-lncRNAs. The DEGs and DE-IncRNAs between the XAV939 group and control group were investigated using the quasi-likelihood F-tests method of the edgeR package (24) in R software (www.r-project.org), with the thresholds of $\mid \log _{2}$ fold-change $(\mathrm{FC}) \mid>0.3$ and $\mathrm{P}<0.05$. The bidirectional hierarchical clustering for DEGs was performed by pheatmap software (v1.0.8; https://cran.r-project. org/web/packages/pheatmap/index.html).

Function and pathway enrichment analysis. The Gene Ontology (GO) database was utilized to identify potential biological processes associated with DEGs, while the Kyoto Encyclopedia of Genes and Genomes (KEGG) database was used to reveal the pathways enriched in DEGs. GO functional categories include molecular function (MF), biological process (BP) and cellular component (CC) (25). The function and pathway enrichment analyses were performed on DEGs using the Database for Annotation, Visualization and Integrated Discovery (DAVID) online tool (26). The cut-off value for a significant function and pathway selection was $\mathrm{P}<0.05$.

Construction of PPI network. To examine the potential interactions of the DEGs, the Search Tool for the Retrieval of Interacting Genes/Proteins database (27) was used to establish the PPI network under the condition of a required confidence (combined score) of $>0.4$. Cytoscape software (version 3.2.1; National Institute of General Medical Sciences, Seattle, WA, USA) (28) was used to visualize the network. A node in the PPI network represents the protein product encoded by DEGs and the degree (Degree Centrality) (29) of a node indicates the number of proteins interacting with this specific node. The top ten nodes ranked by degree (degree $>5$ ) were considered as the hub nodes.

lncRNAs target gene prediction. The correlations between DE-lncRNAs and DEGs were calculated with the Pearson's correlation coefficient (30). The DEGs with Benjamini-Hochberg corrected P-values of $<0.05$ (31) and $|\rho|$ (correlation coefficient) $>0.9$ were considered as potential target genes of IncRNAs.
IncRNA function prediction. GO function and KEGG pathway enrichment analyses were performed on the target genes of DE-lncRNAs to predict the function of the DE-lncRNAs. Using the clusterProfiler package (version 2.2.7; http://www. bioconductor.org/packages/3.1/bioc/html/clusterProfiler.html) in $\mathrm{R}(32), \geq 15$ DE-lncRNA target genes were found and selected for the subsequent analysis. $\mathrm{P}<0.05$ was set as the cut-off value for significant function and pathway enrichment.

Functional similarity analysis of DE-lncRNAs. The Resnik method is an information content-based method (33), while the Wang method is a graph-based method (34). In the present study, the Resnik and Wang methods in the GOSemSim software (version 2.8.0; http://www.bioconductor. org/packages/release/bioc/html/GOSemSim.html) (35) were used to explore the functional similarity among the lncRNAs. A sum of Wang and Resnik methods score $>1.2$, generated by GOSemSim, represented a functional similarity between 2 lncRNAs. The lncRNA functional similarity network was constructed using Cytoscape.

miRNA prediction and ceRNA regulatory network construction. DEG-associated miRNAs were predicted using Enrichr software (2016 version; http://amp.pharm.mssm.edu/Enrichr) with $\mathrm{P}<0.05$ as the threshold (36). Subsequently, the miRanda software (v.3.3a; parameters: -score 120, -energy -20) was used to confirm the regulatory associations between miRNAs and DE-lncRNAs.

Based on the miRNA-DEG, DE-IncRNA-miRNA and DE-lncRNA-DEG interactions, the DEG-miRNA-DE-lncRNA network (ceRNA) was explored further. Finally, the network topology analysis was performed to obtain the key elements in the network.

Statistical analysis. All the OD values were presented as the mean \pm standard deviations (SD). The comparisons between the XAV939-treatment group and control group were analyzed by one-way analysis of variance, followed by the least significant difference test. The SPSS 22.0 software (IBM Corp., Armonk, NY, USA) was used for this analysis, where $\mathrm{P}<0.05$ was considered to indicate a statistically significant difference. 

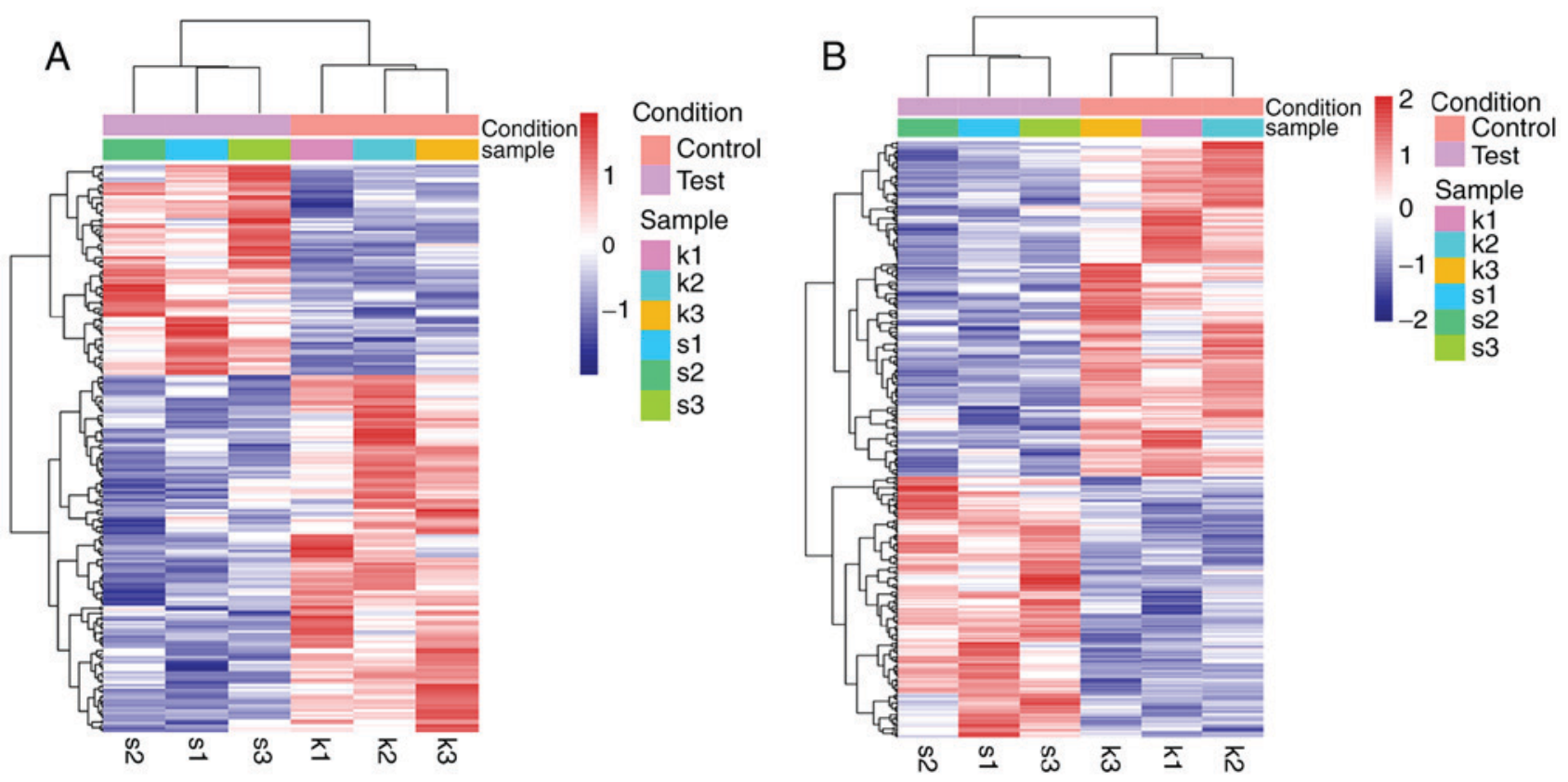

Figure 2. Heatmap for the DEGs and DE-lncRNAs between NCI-H1299 cells in the XAV939 treatment group and in the control group. (A) A heatmap for the DE-lncRNAs. (B) A heatmap for the DEGs. s1-s3 were 3 replicate samples in the XAV939 group; k1-k3 were 3 replicate samples in the control group. Red represents the upregulated genes, while blue represents the downregulated genes. IncRNA, long non-coding RNA; DEG, differentially expressed gene.

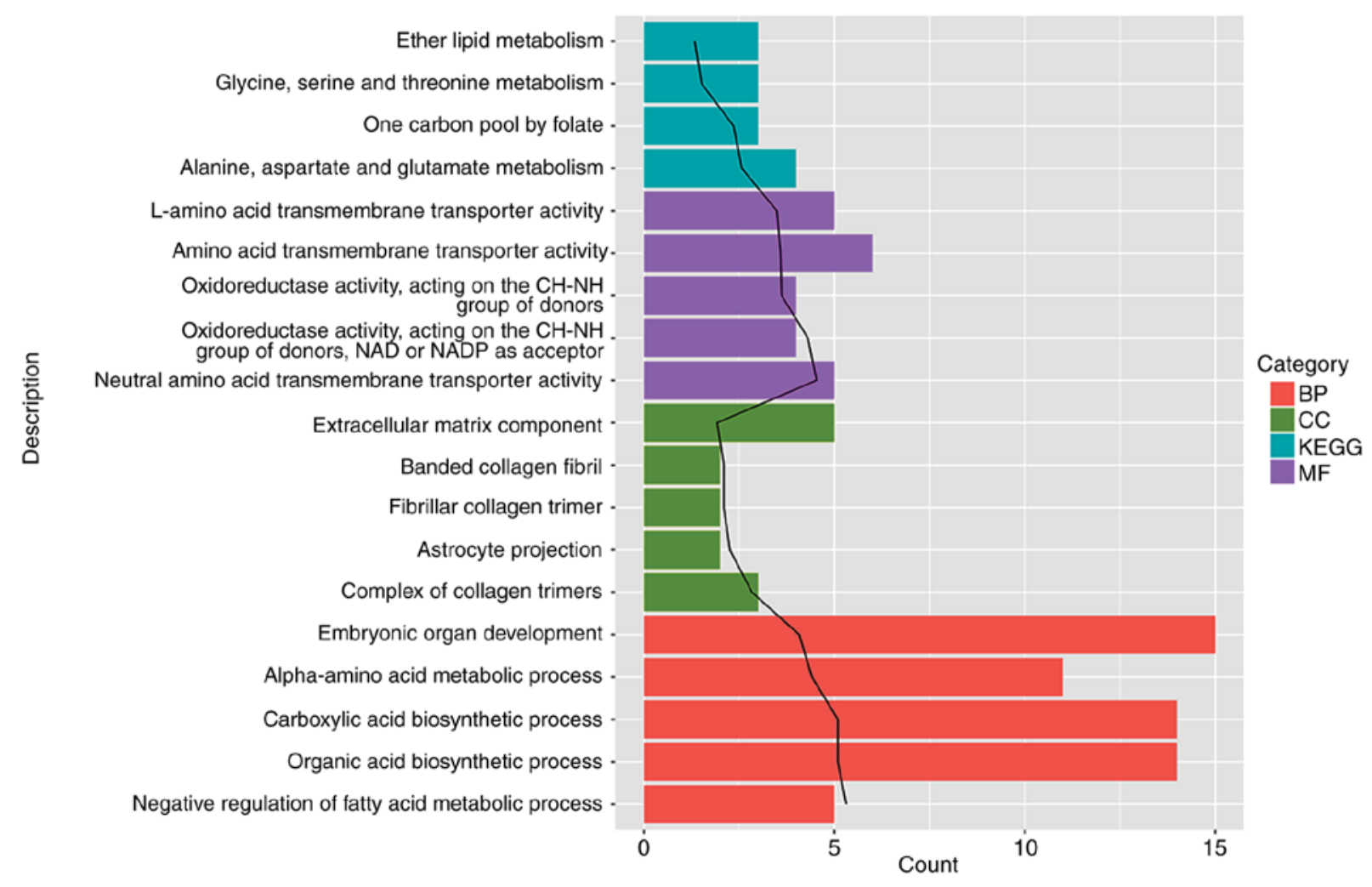

Figure 3. Results of the Gene Ontology function and Koyto Encyclopedia of Genes and Genomes pathway enrichment analyses for the differentially expressed genes, where the top 5 are listed. The black line represents the $-\log _{10}$ (P-value). BP, biological process; CC, cellular components; MF, molecular function; description, the name of the function or pathway; count, the number of genes in a term.

\section{Results}

XAV939 decreases NCI-H1299 cell proliferation, but increases apoptosis. Following treatment with XAV939, the size of NCI-H1299 cells was identified to be slightly smaller under the microscope. The CCK-8 assay was used to determine an appropriate XAV939 concentration that may significantly reduce the cell viability of NCI-H1299 cells compared with other XAV939 concentrations for downstream analysis. The result showed that $10 \mu \mathrm{M}$ XAV939 could significantly inhibit 


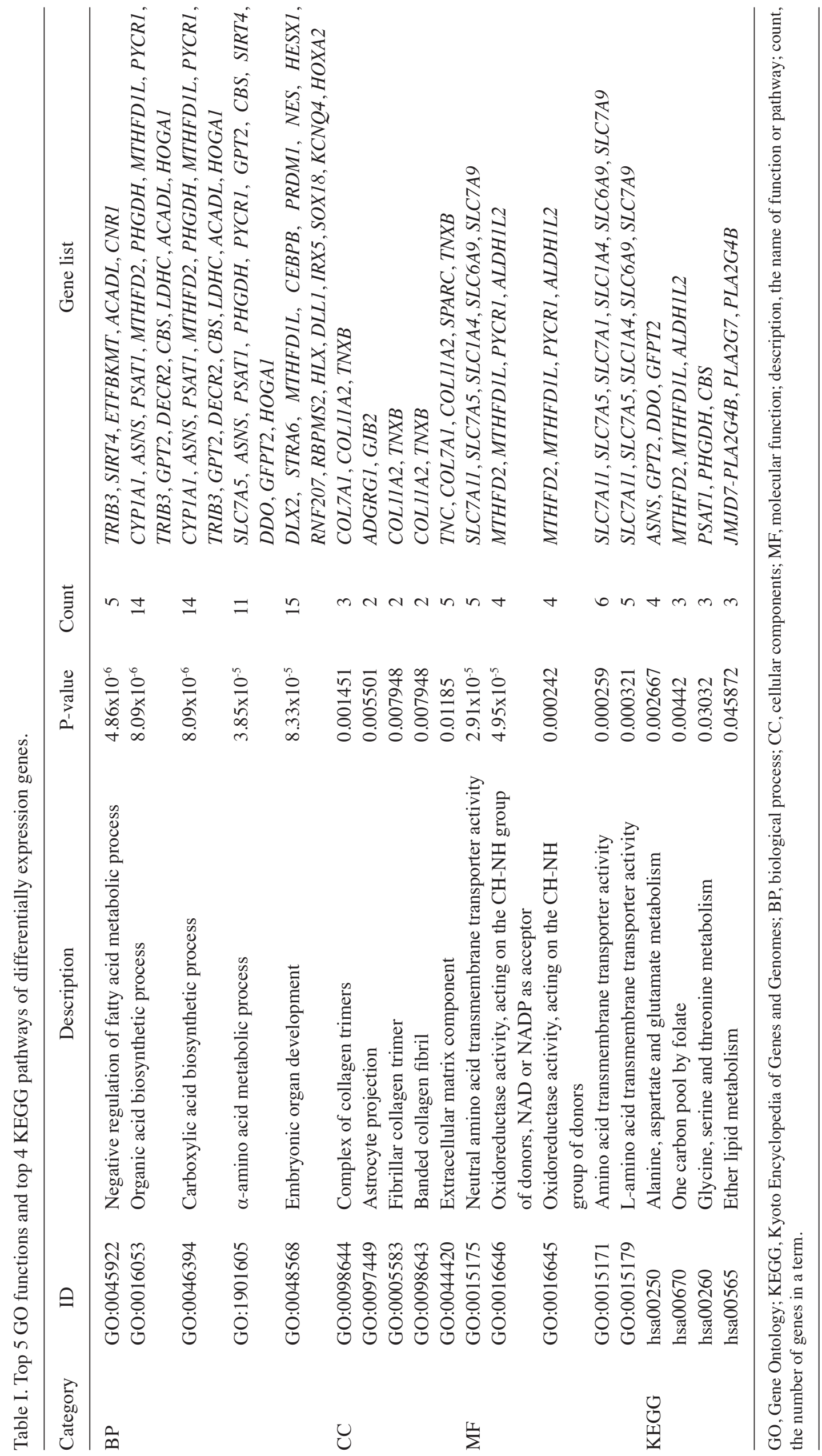




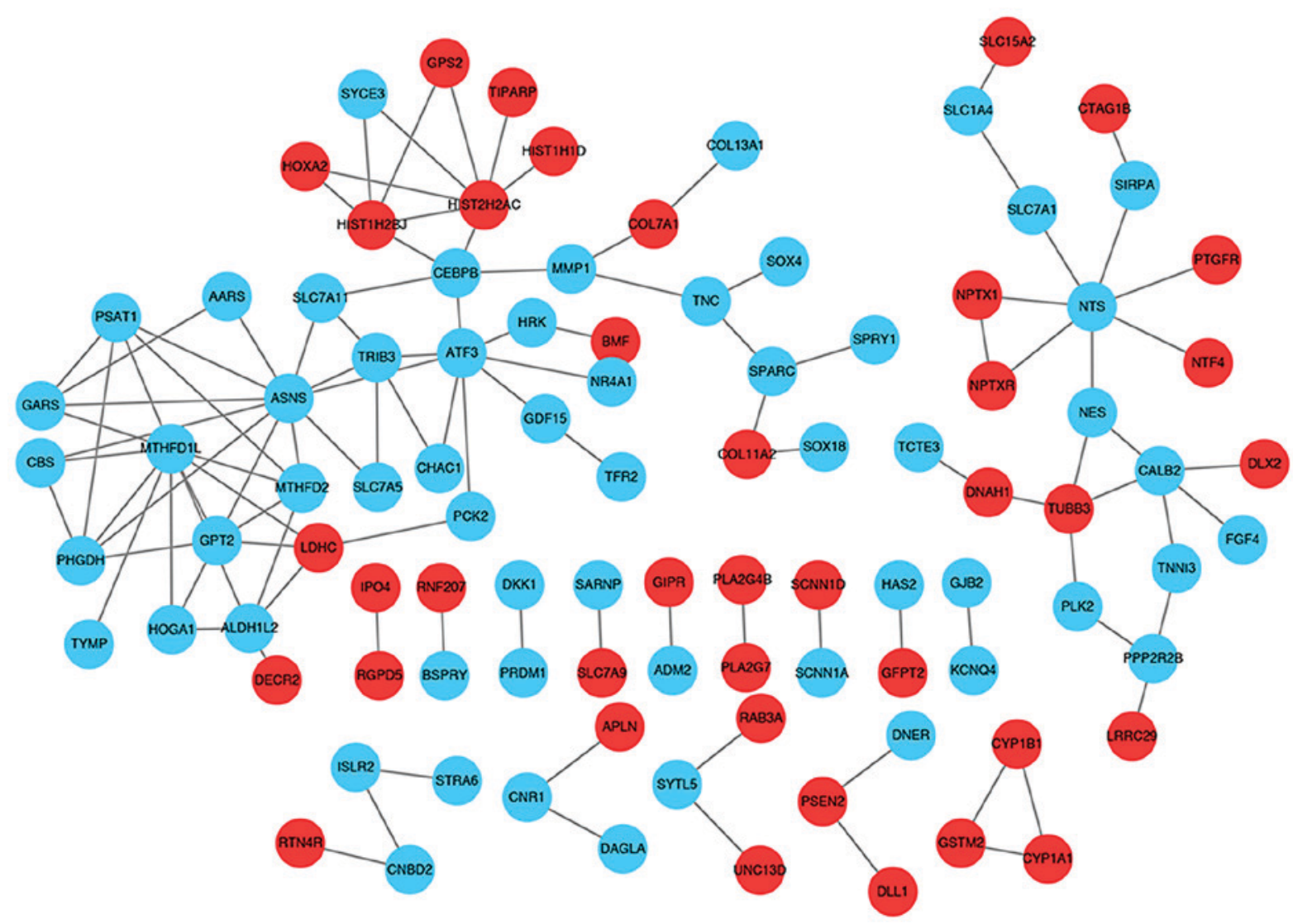

Figure 4. Protein-protein interaction network constructed of differentially expressed genes. Red circles represent the upregulated genes and blue circles represent the downregulated genes.

the proliferation of NCI-H1299 cells $(\mathrm{P}<0.001)$, but no significant differences were identified between the 10 - and $20-\mu \mathrm{M}$ treatment groups $(\mathrm{P}>0.05)$, or between the control and the $20-\mu \mathrm{M}$ treatment group $(\mathrm{P}>0.05 ;$ Fig. $1 \mathrm{~A})$. As a result, $10 \mu \mathrm{M}$ XAV939, which significantly reduced the cell viability of NCI-H1299 cells compared with $20 \mu \mathrm{M}$ XAV939, was used to treat the NCI-H1299 cells in the following investigation. Following this, the apoptosis of the NCI-H1299 cells after treatment with $10 \mu \mathrm{M}$ XAV939 was also determined and revealed to be significantly increased ( $\mathrm{P}=0.004$; Fig. 1B).

DEGs and DE-lncRNAs between XAV939 and control groups. A total of 256 upregulated genes (including 173 DEGs and 83 DE-lncRNAs) and 364 downregulated genes (including 223 DEGs and 141 DE-lncRNAs) were revealed between the XAV939 treatment group and the control group, using the threshold criteria $\log _{2} \mathrm{FCl}>0.3$ and $\mathrm{P}<0.05$. Heatmaps were generated for these DEGs and DE-lncRNAs (Fig. 2).

Enrichment analysis of DEGs. GO functions and KEGG pathways enriched by DEGs were further investigated (Fig. 3; Table I). The results showed that GO DEGs were mainly associated with functions such as 'embryonic organ development' (BP, GO:0048568; $\mathrm{P}=8.33 \times 10^{-5}$ ), 'extracellular matrix component' (CC, GO:0044420; $\mathrm{P}=1.19 \times 10^{-2}$ ) and 'amino acid transmembrane transporter activity' (MF, GO:0015171; $\mathrm{P}=2.59 \times 10^{-4}$ ). Meanwhile, the KEGG DEGs were mainly enriched in pathways such as 'alanine, aspartate and glutamate metabolism' (hsa00250; $\mathrm{P}=2.67 \times 10^{-3}$ ).
PPI network analysis. With a combined score of $>0.4$, a PPI network for the present study was constructed with a total of 97 nodes and 112 interactions (Fig. 4). The top 10 nodes in the PPI network were asparagine synthetase (glutamine-hydrolyzing) (degree=11), methylenetetrahydrofolate dehydrogenase (NADP + dependent) 1 like (degree=10), activating transcription factor 3 (degree=8), neurotensin (degree=7), histone cluster $2 \mathrm{H} 2 \mathrm{~A}$ family member $\mathrm{C}$ (degree=7), glutamic-pyruvic transaminase $2($ degree $=6)$, calbindin $2($ degree $=5)$, tribbles pseudokinase 3 (degree $=5$ ), CCAAT/enhancer binding protein $\beta$ (degree $=5$ ) and aldehyde dehydrogenase 1 family member L2 (degree $=5)$.

DE-lncRNA-target gene interaction. Based on the Pearson's correlation coefficient analysis, a total of 224 DE-lncRNAs had the predicted target genes. Specifically, the top $10 \mathrm{lncRNAs}$ were RP1-101A2.1, RP11-584P21.2, CTD-2246P4.1, ST3 $\beta$-galactosile $\alpha$-2,3-sialyltransferase 6 (ST3GAL6)-antisense RNA 1 ( $A S 1)$, small integral membrane protein 2-AS1, CTD-2553L13.4, AC018766.6, long intergenic non-protein coding RNA (LINC) 926 (LINC00926), RP11-43N16.4 and LINC00973.

$D E-\operatorname{lnc} R N A$ function prediction and comparison. The functional prediction for DE-IncRNAs was obtained via GO and KEGG analyses of target genes of DE-lncRNAs. The results showed that the target genes of DE-IncRNAs were mainly enriched in pathways such as 'ferroptosis' [(DEG; solute carrier family 7 member 11 (SLC7A11)] and tryptophan 


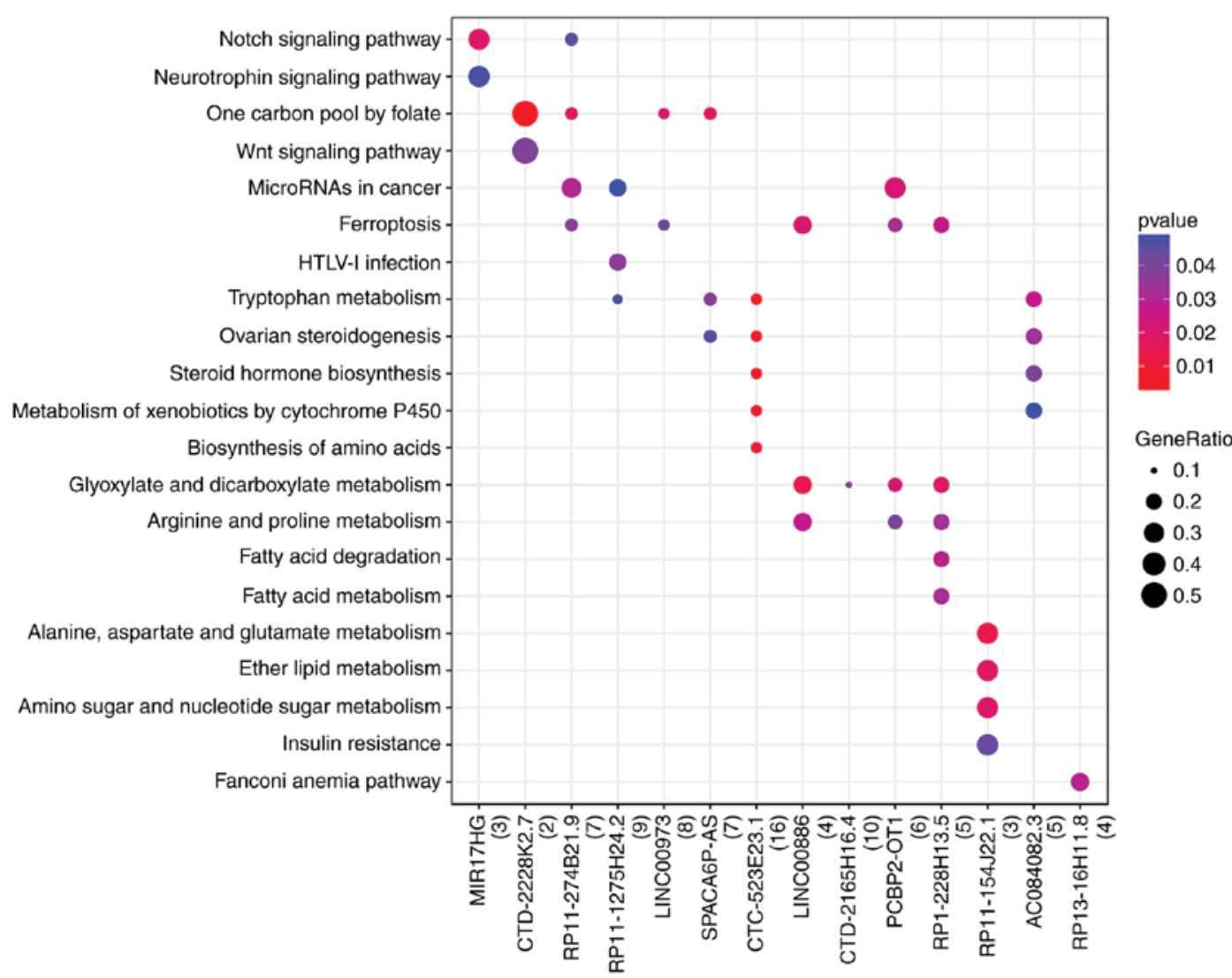

Figure 5. Results of the Kyoto Encyclopedia of Genes and Genomes pathway analysis for lncRNA-associated genes. The P-value key shows the significance of terms enriched by mRNAs; the x-axis represents lncRNAs and the y-axis represents the names of enriched terms. HTLV-1, human T-cell leukemia virus type $1 ;$ lncRNA, long non-coding RNA.

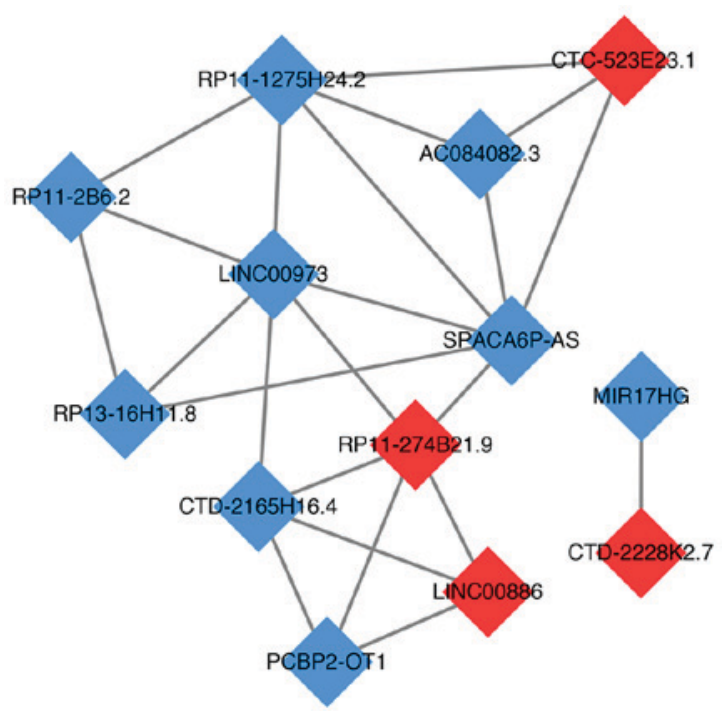

Figure 6. IncRNA functional similarity network. The red squares represent the upregulated IncRNAs, while the blue squares represent the downregulated lncRNAs. lncRNA, long non-coding RNA.

metabolism' (DEG; cytochrome p450 family 1 subfamily B member 1 (Fig. 5).

DE-lncRNA functional similarity. The DE-IncRNA functional similarity network was constructed using Cytoscape software (Fig. 6). The results showed that there were 9 downregulated lncRNAs, including LINC00973, sperm acrosome-associated 6 antisense RNA (SPACA6P-AS), AC084082.3, RP11-1275H24.2, RP11-2B6.2, RP13-16H11.8, $C T D-2165 H 16.4$, poly (rC) binding protein 2-overlapping transcript 1 and miR-17-92a-1 cluster host gene, and 4 upregulated lncRNAs, including CTC-523E23.1, CTD-2228K2.7, RP11-274B21.9 and LINC00886, in this network. Among these IncRNAs, LINC00973 and SPACA6P-AS were 2 noteworthy DE-lncRNAs that had the highest number of interactions.

ceRNA regulatory network investigation. Based on the miRNA-DEG interactions (Table II), the DE-lncRNA-miRNA interactions and the DE-lncRNA-DEG interactions, the ceRNA network was further explored (Fig. 7). There were 18 upregulated DEGs [including distal-less homeobox 2 (DLX2)], 30 downregulated DEGs [including SRY-box 4 (SOX4)], 4 upregulated IncRNAs (including LINC01419), 13 downregulated lncRNAs [including MIR503 host gene (MIR503HG)] and 18 miRNAs (including hsa-miR-1273c) in this ceRNA network. The results identified several miRNA-lncRNA-DEG associations, including MIR503HG-miR1273c-SOX4 and ST3GAL6-AS1-miR4669-DLX2, which were of note in the ceRNA network.

\section{Discussion}

Worldwide, NSCLC is one of the most lethal cancer affecting individuals (37). Understanding the molecular mechanisms of 


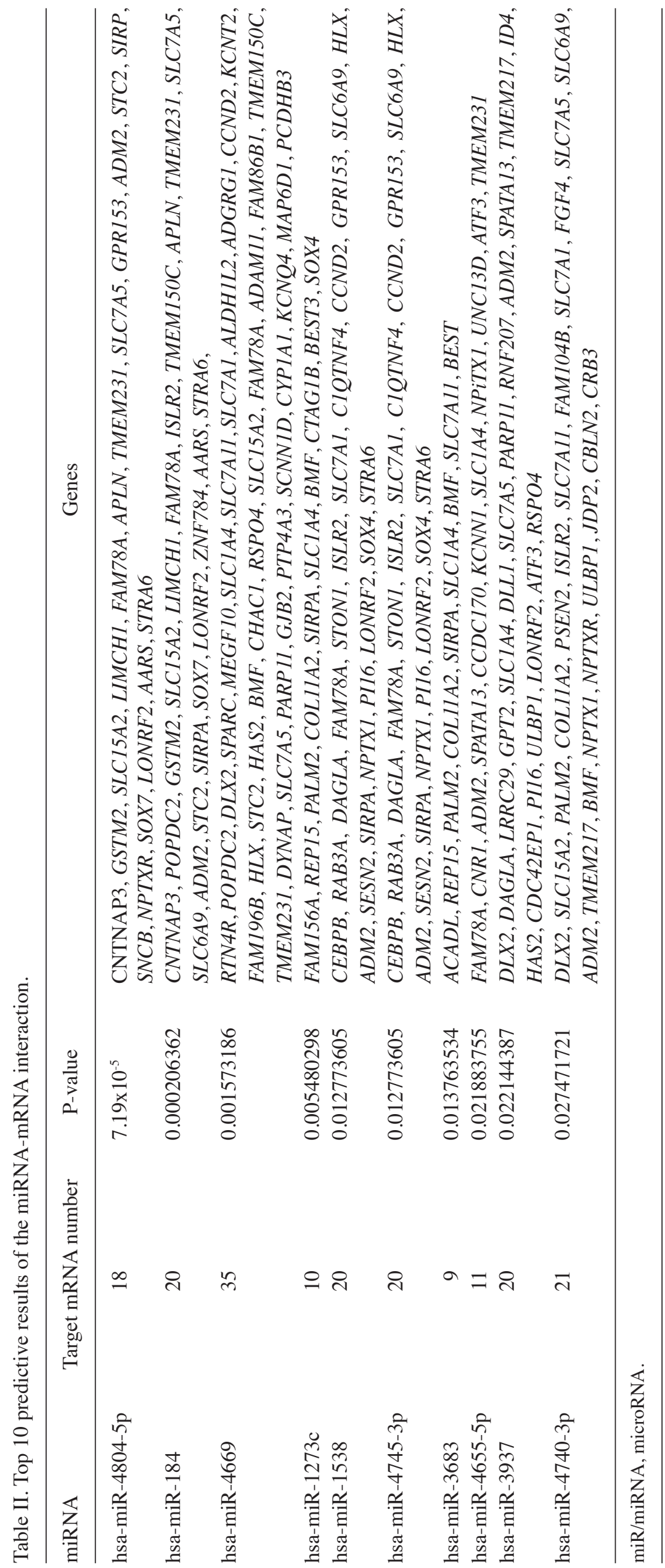




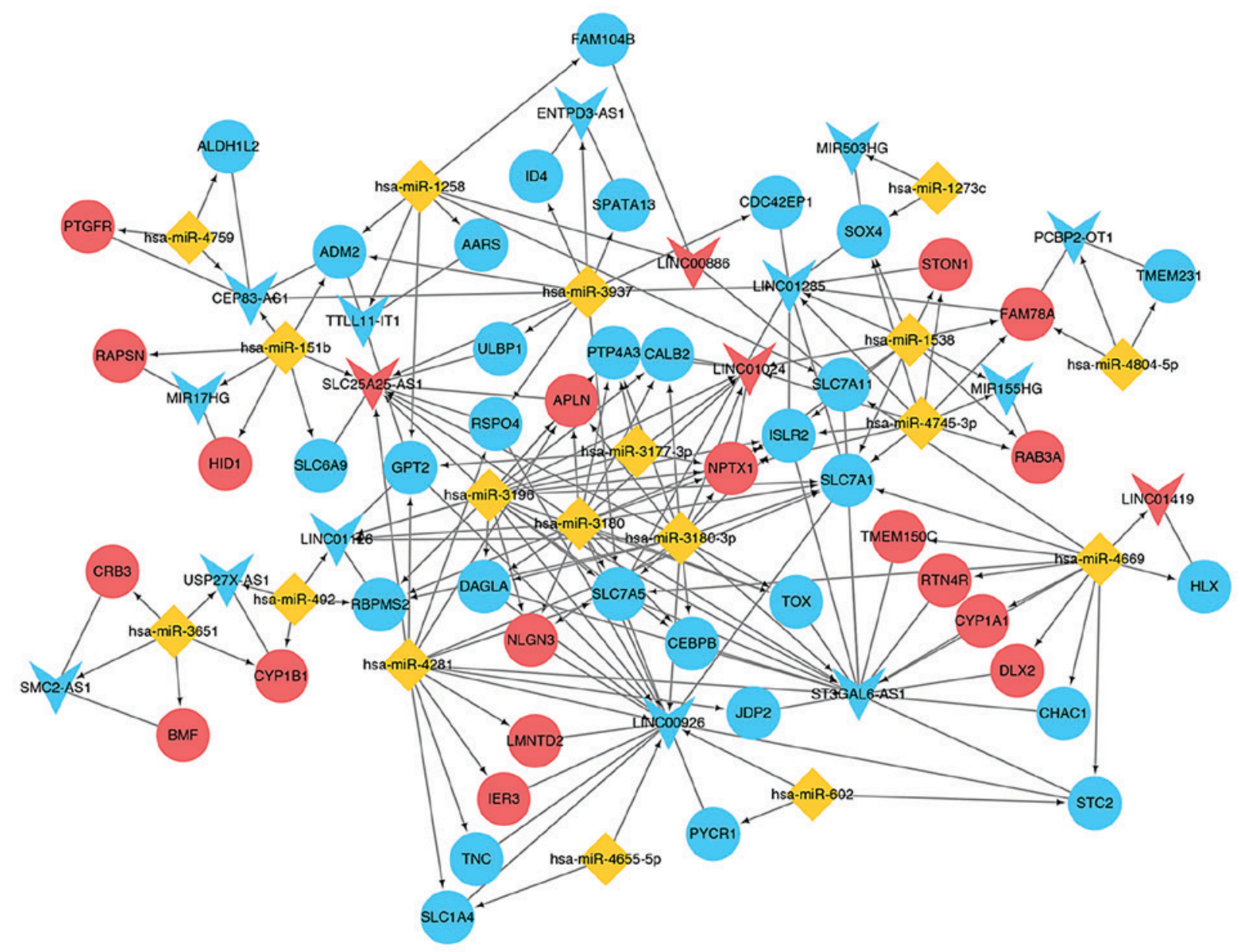

Figure 7. Competing endogenous RNA network constructed by miRNAs, lncRNAs and mRNAs. Yellow diamonds represent miRNAs; blue triangles represent downregulated lncRNAs; red triangles represent upregulated lncRNAs; blue circles represent downregulated mRNAs; red circles represent upregulated mRNAs; the straight line represents lncRNA-mRNA interaction; the arrow represents the miRNA-lncRNA or miRNA-mRNA associations. lncRNA, long non-coding RNA; miRNA, microRNA.

NSCLC may contribute to the therapeutic clinical outcomes in patients. In the present study, a total of 396 DEGs and 224 DE-lncRNAs were identified in the NSCLC NCI-H1299 cell line between the XAV939 treatment and control groups. These lncRNAs included pathways such as the 'ferroptosis' pathway (DEG, SLC7A11). Furthermore, the lncRNA-target interaction investigation revealed $10 \mathrm{lncRNAs}$ that were noteworthy, including ST3GAL6-AS1. Finally, the ceRNA network identified several novel miRNA-lncRNA-mRNA regulatory associations, including MIR503HG-miR1273c-SOX4.

XAV939 can inhibit the proliferation of carcinoma cells by repressing specific gene expression, including that of lactate dehydrogenase A (38). In the present study, the ceRNA network analysis showed that $M I R 503 H G$, which was inhibited by XAV939, was one of the most significantly downregulated lncRNAs correlated with the upregulation of $m i R 1273 \mathrm{c}$ and downregulation of SOX4. Importantly, IncRNAs have recently been reported in tumorigenesis and serve a pivotal role in regulating cell cycle behavior (39). A study by Muys et al (40) showed that the IncRNA MIR503HG impairs the migration and invasion capacities in a choriocarcinoma tumor cell, indicating a potential role in human reproduction and tumorigenesis. Knockdown of MIR503HG leads to a strong downregulation of the target gene collagen type $1 \alpha 1$ chain, indicating an important role of $M I R 503 H G$ in diseases, including systemic sclerosis (41). As the lncRNAs target genes, mRNA expression is commonly involved in the pathogenesis and prognosis of lung cancer (42). SOX4 is a critical developmental transcription factor in vertebrates (43). A previous study indicated that a SOX4 gene mutation is associated with lung carcinogenesis and tumor metastasis (44). By targeting SOX4, miR-212 functions as a tumor suppressor in the metastasis of NSCLC (45). In addition, Li et al (46) reported that silencing SOX4 can inhibit the migration and invasion of tumor cells in the lung. Actually, miRNAs can be used as biomarkers for NSCLC (47). Lee et al (48) indicated that $m i R 1273 c$, a member of the miR1273 family, serves an inhibitory role in the progression of cancer cells. However, the regulatory effect of $m i R 1273 c$ on NSCLC remains unclear. The present study indicated that MIR503HG-miR1273c-SOX4 was one of the notable interactions in the ceRNA network. Thus, we hypothesize that the downregulation of $\operatorname{lncRNA} M I R 503 H G$ induced by XAV939 may serve an important role in inhibiting the progression of NSCLC by sponging $m i R-1273 c$ and downregulating SOX4 expression.

A previous study showed that XAV939 can be used as an agent for lung cancer therapy (17). The inhibition of XAV939 in the proliferation and migration of lung adenocarcinoma cells was determined by modulating certain biological 
pathways, including that of WNT (49). Ferroptosis, a type of programmed cell death dependent on iron, inhibits tumor growth and increases drug resistance $(50,51)$. A previous study revealed that the inhibition of ferroptosis influences lung cancer progression (52). Although the association between the ferroptosis pathway and lung cancer progression has already been proved (53), the detailed mechanism of ferroptosis on the development of NSCLC remains unclear. The solute carrier family (SLF) is a group of membrane transport proteins located in the cell membrane (54). As a member of the SLF family, SLC7A11 contributes to the temozolomide toxicity via the ferroptosis pathway (55). A previous study revealed that tumor suppression occurred by repression of SLC7A11 in the ferroptosis pathway (56). More importantly, the upregulation of SLC7A11 in transformed airway epithelial cells was able to induce tumor formation in nude mice (57). In the present study, the lncRNA function analysis revealed that the ferroptosis pathway, which was associated with SLC7A11, was one of the significant pathways affected. In addition, the results revealed that SLC7A11 was downregulated in XAV939-treated NCI-H1299 cells. Collectively, these results indicate that the downregulation of SLC7A11 induced by XAV939 may suppress the development of NSCLC via the ferroptosis pathway.

However, there are limitations in the present study, including the use of a single cell line and lack of a verification test. Thus, a larger sample size using additional cell lines, with a wide verification analysis is required in future investigations. In addition, the dose and time point selected were used as referenced by similar studies, and a lack of dose-dependent and time-dependent experiments is therefore also a limitation of the present study. The present study was preliminary and several important bioinformatics results, including expression changes and regulatory mechanisms discussed, require validation by biological experiments in future studies.

In conclusion, the downregulation of the IncRNA MIR503HG induced by XAV939 may serve an important role in suppressing the progression of NSCLC via sponging $m i R 1273 \mathrm{c}$, to downregulate its target SOX4. Furthermore, the downregulation of SLC7A11 induced by XAV939 may inhibit NSCLC development via participation in the ferroptosis pathway.

\section{Acknowledgements}

Not applicable.

\section{Funding}

The present study was supported by The Study of the Correlation Between ROCK1 and the Metastasis of Lung Cancer (grant no. SCZSY201728).

\section{Availability of data and materials}

The datasets used and/or analyzed during the present study are available from the corresponding author on reasonable request.

\section{Authors' contributions}

$\mathrm{HX}$, LX and HY were responsible for the conception and design of the study, and drafted the manuscript. $\mathrm{ZH}$ and $\mathrm{ZX}$ performed the data acquisition. HY and CA performed the data analysis and interpretation. LX and HY performed the statistical analysis. All authors have read and approved the manuscript.

\section{Ethical approval and consent to participate}

Not applicable.

\section{Patient consent for publication}

Not applicable.

\section{Competing interests}

The authors declare that they have no competing interests.

\section{References}

1. Johnson BE: Divide and conquer to treat lung cancer. N Engl J Med 375: 1892-1893, 2016.

2. Chen Z, Fillmore CM, Hammerman PS, Kim CF and Wong KK: Non-small-cell lung cancers: A heterogeneous set of diseases. Nat Rev Cancer 14: 535-546, 2014.

3. Siegel R, Naishadham D and Jemal A: Cancer statistics, 2013. CA Cancer J Clin 63: 11-30, 2013.

4. Schiller JH, Harrington D, Belani CP, Langer C, Sandler A, Krook J, Zhu J and Johnson DH; Eastern Cooperative Oncology Group: Comparison of four chemotherapy regimens for advanced non-small-cell lung cancer. N Engl J Med 346: 92-98, 2002.

5. Petty RD, Nicolson MC, Kerr KM, Collie-Duguid E and Murray GI: Gene expression profiling in non-small cell lung cancer: From molecular mechanisms to clinical application. Clin Cancer Res 10: 3237-3248, 2004.

6. Cheng N, Li X, Zhao C, Ren S, Chen X, Cai W, Zhao M, Zhang Y, Li J, Wang Q and Zhou C: Microarray expression profile of long non-coding RNAs in EGFR-TKIs resistance of human non-small cell lung cancer. Oncol Rep 33: 833-839, 2015.

7. Wilusz JE, Sunwoo H and Spector DL: Long noncoding RNAs: Functional surprises from the RNA world. Genes Dev 23: 1494-1504, 2009

8. Zhou M, Guo M, He D, Wang X, Cui Y, Yang H, Hao D and Sun J: A potential signature of eight long non-coding RNAs predicts survival in patients with non-small cell lung cancer. J Transl Med 13: 231, 2015

9. Ghadimi K, Bahrami N, Fathi M, Farzanegan B, Naji T, Emami M and Mohamadnia A: Diagnostic value of LunX mRNA and CEA mRNA expression in pleural fluid of patients with non-small cell lung cancer. Minerva Pneumol 56: 90-95, 2017

10. Zhou HX, Yang MX, Wang Y, Cao WM, Lu KF, Zong LY, Wu RQ and Zhang P: Plasma LUNX mRNA, a non-invasive specific biomarker for diagnosis and prognostic prediction of non-small cell lung cancer. Am J Cancer Res 6: 452-458, 2016.

11. Xu C, Xu Y, Liao X, Liao R, Zhang L, Niu K, Li T, Li D, Chen Z, Duan $Y$ and Sun J: Plasma miRNAs in predicting radiosensitivity in non-small cell lung cancer. Tumour Biol 37: 11927-11936, 2016.

12. Wu C and Arora P: Long noncoding RNA-microRNA-mRNA: A novel tripartite axis in the regulation of cardiac hypertrophy. Circ Cardiovasc Genet 7: 729-731, 2014.

13. Ye S, Yang L, Zhao X, Song W, Wang W and Zheng S: Bioinformatics method to predict two regulation mechanism: TF-miRNA-mRNA and lncRNA-miRNA-mRNA in pancreatic cancer. Cell Biochem Biophys 70: 1849-1858, 2014.

14. Yang ZH, Zheng R, Gao Y, Zhang Q and Zhang H: Abnormal gene expression and gene fusion in lung adenocarcinoma with high-throughput RNA sequencing. Cancer Gene Ther 21: 74-82, 2014.

15. Leidinger P, Brefort T, Backes C, Krapp M, Galata V, Beier M, Kohlhaas J, Huwer H, Meese E and Keller A: High-throughput qRT-PCR validation of blood microRNAs in non-small cell lung cancer. Oncotarget 7: 4611-4623, 2016.

16. Tian X, Hou W, Bai S, Fan J, Tong H and Bai Y: XAV939 promotes apoptosis in a neuroblastoma cell line via telomere shortening. Oncol Rep 32: 1999-2006, 2014. 
17. Guo W, Shen F, Xiao W, Chen J and Pan F: Wnt inhibitor XAV939 suppresses the viability of small cell lung cancer NCI-H446 cells and induces apoptosis. Oncol Lett 14: 6585-6591, 2017.

18. Lin HH, Feng WC, Lu LC, Shao YY, Cheng AL and Hsu CH: Abstract 2052: WNT/beta-catenin signaling inhibitors improve the anti-proliferative effect of sorafenib against hepatocellular carcinoma (HCC) cells. Cancer Res 73: 2052, 2013.

19. Liu Y, Su D and Song T: Programmed cell death 4 inhibits proliferation and differentiation and induces apoptosis of human mesenchymal stem cells through suppressing the Wnt/ $\beta$-catenin pathway. RSC Adv 7: 26566-26573, 2017.

20. Ran M, Chen B, Li Z, Wu M, Liu X, He C, Zhang S and Li Z: Systematic identification of long non-coding RNAs in immature and mature porcine testes. Biol Reprod 94: 77, 2016.

21. Bolger AM, Lohse M and Usadel B: Trimmomatic: A flexible trimmer for Illumina sequence data. Bioinformatics 30 : 2114-2120, 2014.

22. Harrow J, Frankish A, Gonzalez JM, Tapanari E, Diekhans M, Kokocinski F, Aken BL, Barrell D, Zadissa A, Searle S, et al: GENCODE: The reference human genome annotation for The ENCODE Project. Genome Res 22: 1760-1774, 2012.

23. Trapnell C, Pachter L and Salzberg SL: TopHat: Discovering splice junctions with RNA-Seq. Bioinformatics 25: 1105-1111, 2009.

24. Lun AT, Chen Y and Smyth GK: It's DE-licious: A recipe for differential expression analyses of RNA-seq experiments using quasi-likelihood methods in edgeR. Methods Mol Biol 1418 391-416, 2016.

25. Gene Ontology Consortium: The gene ontology in 2010 : Extensions and refinements. Nucleic Acids Res 38(Database Issue): D331-D335, 2010.

26. Huang DW, Sherman BT, Tan Q, Kir J, Liu D, Bryant D, Guo Y, Stephens R, Baseler MW, Lane HC and Lempicki RA: DAVID bioinformatics resources: Expanded annotation database and novel algorithms to better extract biology from large gene lists. Nucleic Acids Res 35 (Web Server Issue): W169-W175, 2007.

27. Szklarczyk D, Franceschini A, Wyder S, Forslund K, Heller D, Huerta-Cepas J, Simonovic M, Roth A, Santos A, Tsafou KP, et al STRING v10: Protein-protein interaction networks, integrated over the tree of life. Nucleic Acids Res 43 (Database Issue) D447-D452, 2015.

28. Shannon P, Markiel A, Ozier O, Baliga NS, Wang JT, Ramage D, Amin N, Schwikowski B and Ideker T: Cytoscape: A software environment for integrated models of biomolecular interaction networks. Genome Res 13: 2498-2504, 2003.

29. Opsahl T, Agneessens F and Skvoretz J: Node centrality in weighted networks: Generalizing degree and shortest paths. Social Networks 32: 245-251, 2010.

30. Pearson K: Note on regression and inheritance in the case of two parents. Proc R Soc Lond 58: 240-242, 1895.

31. Benjamini $\mathrm{Y}$ and Hochberg Y: Controlling the false discovery rate: A practical and powerful approach to multiple testing. J R Statist Soc 57: 289-300, 1995.

32. Yu G, Wang LG, Han Y and He QY: clusterProfiler: An R package for comparing biological themes among gene clusters. OMICS 16: 284-287, 2012

33. Resnik P: Semantic similarity in a taxonomy: An information-based measure and its application to problems of ambiguity in natural language. J Artif Intell Res 11: 95-130, 1999.

34. Wang JZ, Du Z, Payattakool R, Yu PS and Chen CF: A new method to measure the semantic similarity of GO terms. Bioinformatics 23: 1274-1281, 2007.

35. Yu G, Li F, Qin Y, Bo X, Wu Y and Wang S: GOSemSim: An R package for measuring semantic similarity among GO terms and gene products. Bioinformatics 26: 976-978, 2010.

36. Kuleshov MV, Jones MR, Rouillard AD, Fernandez NF, Duan Q, Wang Z, Koplev S, Jenkins SL, Jagodnik KM, Lachmann A, et al: Enrichr: A comprehensive gene set enrichment analysis web server 2016 update. Nucleic Acids Res 44 (W1): W90-W97, 2016.

37. Zhan Y, Zang H, Feng J, Lu J, Chen L and Fan S: Long non-coding RNAs associated with non-small cell lung cancer. Oncotarget 8 : 69174-69184, 2017.

38. Zheng K, Wu G, Qin X, Wang Y, Xia S and Meng X: Effects of XAV939 on proliferation and glycolysis of hepatocellular carcinoma cells. J Mod Oncol 13: 2023-2026, 2016.

39. Teng H, Wang P, Xue Y, Liu X, Ma J, Cai H, Xi Z, Li Z and Liu Y: Role of HCP5-miR-139-RUNX1 feedback loop in regulating malignant behavior of glioma cells. Mol Ther 24: 1806-1822, 2016
40. Muys BR, Lorenzi JC, Zanette DL, Lima e Bueno Rde B de Araújo LF, Dinarte-Santos AR, Alves CP, Ramão A, de Molfetta GA, Vidal DO and Silva WA Jr: Placenta-Enriched LincRNAs MIR503HG and linc00629 decrease migration and invasion potential of JEG-3 cell line. PLoS One 11: e0151560, 2016.

41. Pachera E, Assassi S, Cintora GS, Frank-Bertoncelj M, Haunerdinger V, Dobrota R, Brock M, Vettori S, Hellerbrand C Feghali-Bostwick CA, et al: OP0284 long noncoding RNA MIR503HG is a novel factor in the pathogenesis of systemic sclerosis. Ann Rheum Dis 74 (Suppl 2): S180.1-180, 2015.

42. Grimminger PP, Maus MK, Schneider PM, Metzger R, Hölscher AH, Sugita H, Danenberg PV, Alakus H and Brabender J: Glutathione S-transferase PI (GST-PI) mRNA expression and DNA methylation is involved in the pathogenesis and prognosis of NSCLC. Lung Cancer 78: 87-91, 2012.

43. Scharer CD, Mccabe CD, Aliseyed M, Berger MF, Bulyk ML and Moreno CS: Genome-wide promoter analysis of the SOX4 transcriptional network in prostate cancer cells. Cancer Res 69: 709-717, 2009.

44. Chen QL, Zheng WL, Yao WJ, Nie LW, Cheng SH and Ma WL: Analysis of SOX 4 gene mutation in non-small cell lung cancer tissues. Zhonghua Yi Xue Yi Chuan Xue Za Zhi 24: 505-509, 2007.

45. Tang T, Huan L, Zhang S, Zhou H, Gu L, Chen X and Zhang L: MicroRNA-212 functions as a tumor-suppressor in human non-small cell lung cancer by targeting SOX4. Oncol Rep 38: 2243-2250, 2017.

46. Li Y,Chen P,Zu L, Liu B, Wang M and Zhou Q: MicroRNA-338-3p suppresses metastasis of lung cancer cells by targeting the EMT regulator Sox4. Am J Cancer Res 6: 127-140, 2016.

47. Foss KM, Sima C, Ugolini D, Neri M, Allen KE and Weiss GJ: miR-1254 and miR-574-5p: Serum-based microRNA biomarkers for early-stage non-small cell lung cancer. J Thorac Oncol 6: 482-488, 2011.

48. Lee CW, Kang MR, Yun J, Oh SJ and Kang JS: Abstract 4387: Up-regulation of VHL by miR-1273C inhibits renal cell carcinoma. Cancer Res 74: 4387, 2014.

49. Li C, Zheng X, Han Y, Lv Y, Lan F and Zhao J: XAV939 inhibits the proliferation and migration of lung adenocarcinoma A549 cells through the WNT pathway. Oncol Lett 15: 8973-8982, 2018.

50. Lachaier E, Louandre C, Ezzoukhry Z, Godin C, Mazière JC, Chauffert B and Galmiche A: Ferroptosis, a new form of cell death relevant to the medical treatment of cancer. Med Sci (Paris) 30: 779-783, 2014 (In French).

51. Dixon SJ, Lemberg KM, Lamprecht MR, Skouta R, Zaitsev EM, Gleason CE, Patel DN, Bauer AJ, Cantley AM, Yang WS, et al: Ferroptosis: An iron-dependent form of nonapoptotic cell death. Cell 149: 1060-1072, 2012.

52. Jiang Y, He Y, Liu S and Tao Y: Chromatin remodeling factor lymphoid-specific helicase inhibits ferroptosis through lipid metabolic genes in lung cancer progression. Chin J Cancer 36: 82, 2017.

53. Alvarez SW, Sviderskiy VO, Terzi EM, Papagiannakopoulos T, Moreira AL, Adams S, Sabatini DM, Birsoy K and Possemato R: NFS1 undergoes positive selection in lung tumours and protects cells from ferroptosis. Nature 551: 639-643, 2017.

54. He L, Vasiliou K, and Nebert DW: Analysis and update of the human solute carrier (SLC) gene superfamily. Hum Genomics 3: 195-206, 2009.

55. Sehm T, Rauh M, Wiendieck K, Buchfelder M, Eyüpoglu IY and Savaskan NE: Temozolomide toxicity operates in a xCT/SLC7a11 dependent manner and is fostered by ferroptosis. Oncotarget 7: 74630-74647, 2016.

56. Jiang L: p53 promotes ferroptosis during ROS stress to suppress tumorigenesis. Cancer Dis 5: 465, 2015.

57. Ji XJ, Qian J, Rahman J, Harris B, Hoeksema MD, Chen H, Eisenberg R and Young J: Abstract A10: SLC7A11 contributes to the pathogenesis of lung cancer. Mol Cancer Res 14: A10, 2016.

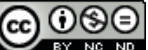

This work is licensed under a Creative Commons Attribution-NonCommercial-NoDerivatives 4.0 International (CC BY-NC-ND 4.0) License. 